\title{
RHEOLOGICAL SLS MODEL. DYNAMIC PARAMETERS OF THE MECHANICAL SYSTEMS WITH VISCOUS DAMPING. PART 1: AMPLITUDE FACTOR
}

\author{
Assoc. Prof. Dr. Eng. Nicusor DRAGAN \\ MECMET - The Research Center of Machines, \\ Mechanic and Technological Equipments \\ Engineering and Agronomy Faculty of \\ Braila \\ "Dunarea de Jos" University of Galati
}

\begin{abstract}
The article presents the dynamic model of a 1DOF elastic system with viscous damping, perturbed by a harmonic force $F(t)=H$ s i nost. The rheological model of the damping is Standard Linear Solid (SLS) model, the analyzed parameter being the amplitude factor/ratio, the transmissibility factor and the isolation degree. This study, which analyzes the amplitude factor $A(\Omega, \zeta)$ function of pulsation/frequency ratio $\Omega$ and the viscous damping ratio $\zeta$, is useful to validate and/or to assess the viscous materials with SLS model behavior.
\end{abstract}

KEYWORDS: steady-state vibration, viscous damping, SLS model, amplitude factor

\section{INTRODUCTION. SLS RHEOLOGICAL MODEL}

The books on viscoelasticity describe basic linear rheological models and complex rheological models [1] [2]. Figure 1 shows the models of linear spring (Hooke) and linear dashpot (Newton).

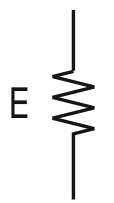

a)

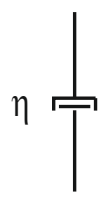

b)
Fig. 1. Basic rheological elements [1] [2]

a) Hooke model

b) Newton model

By combining in series and/or in parallel springs and dashpots, we can obtain complex rheological models. Figure 2 shows the rheological viscoelastic models Maxwell and Kelvin-Voigt, which are the simplest linear viscoelastic models [3] [4] [5].

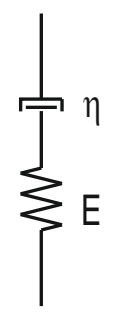

a)

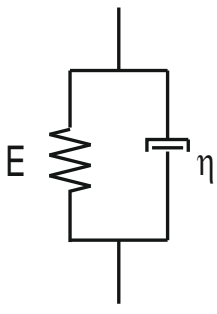

b)
Fig. 2. Linear rheological models [3] [4] [5]

a) serial spring+dashpot (Maxwell model)

b) parallel spring+dashpot (Kelvin-Voigt model)

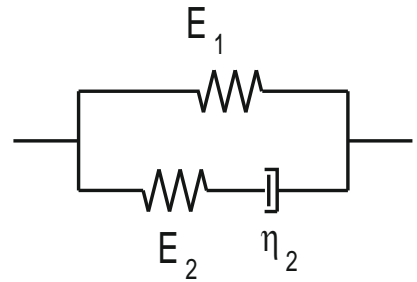


Fig. 3. Standard Linear Solid model [6] (parallel Hooke+Maxwell)

Figure 3 shows the SLS rheological model, which is a parallel combination of Hooke model and Maxwell model. The SLS model can describe, at the same time, the stress relaxation and creep or recovery of a viscous/elastic mechanical system under a given set of loading conditions.

\section{DOF MECHANICAL SYSTEM.} SLS VISCOUS DAMPING MODEL

Figure 4 shows the simplified model of a $1 \mathrm{DOF}$ mechanical system. The mass $\mathrm{m}$ is borne on the viscoelastic support VEM, modeled as a complex rheological element. If the mass $\mathrm{m}$ is perturbed by a variable force $F(t)$, the vertical displacement is $z_{f}(t)$. The rheological characteristics of the VEM determine the dynamic parameters: displacement $z_{f}(t)$ and transmitted force $F_{T}(t)$.

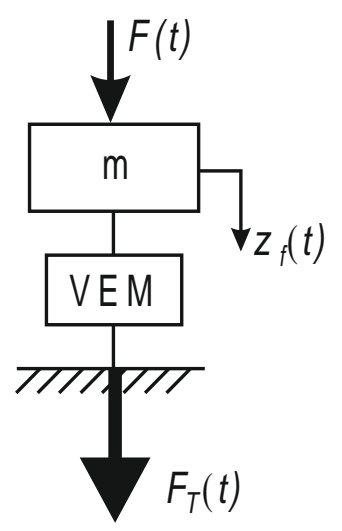

Fig. 4. Model of 1DOF viscoelastic mechanical system [3] [4] [5]

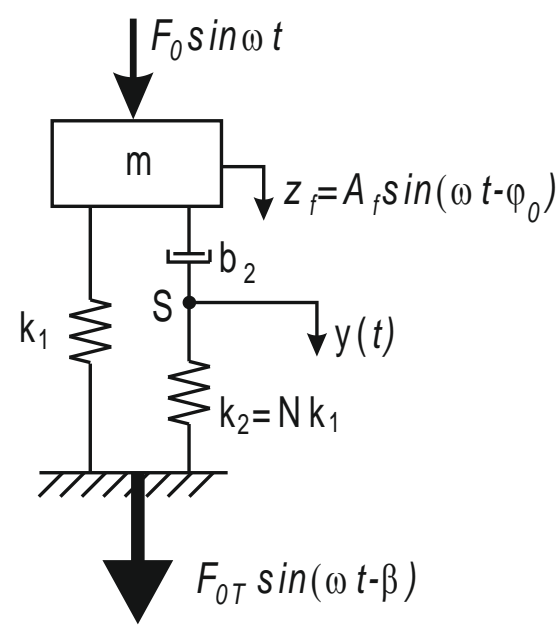

Fig. 5. Model of 1DOF mechanical system with viscous damping (SLS rheological model) perturbed by a harmonic force [3] [4] [5]

Figure 5 shows the calculus model of 1DOF mechanical system with SLS viscous damping, where:

- $k_{1}$ is the stiffness coefficient (Hooke model)

- $\left(k_{2}, b_{2}\right)$ are the stiffness and viscous damping coefficients (Maxwell model)

- $F(t)=F_{0} s$ i $m t$ is the perturbation harmonic force

$-F_{T}(t)=F_{0 T} s(a b t-\beta)$ is the transmitted force to the base

$z_{f}(t)=A_{f} s \quad(\omega t-\alpha)$ is the displacement of the mass $m$

\section{DYNAMIC ANALYSIS OF 1DOF \\ MECHANICAL SYSTEM. SLS VISCOUS DAMPING}

The dynamic equations of the $1 \mathrm{DOF}$ mechanical system from figure 5 are [6]:

$$
\left\{\begin{array}{c}
m \ddot{z}+b_{2}\left(\dot{z}_{f}-\dot{y}\right)+k_{1} z_{f}=F_{0} s \quad \dot{a b t} \\
b_{2}\left(\dot{z}_{f}-\dot{y}\right)=k_{2} y
\end{array}\right.
$$

Dividing by $m$ the first equation of system (1), we obtain

$$
\ddot{z}_{f}+\frac{b_{2}}{m}\left(\dot{z}_{f}-\dot{y}\right)+\frac{k}{m} z_{f}=\frac{F_{0}}{m} s \quad \omega i t
$$

or

$$
\ddot{z}_{f}+2 n\left(\dot{z}_{f}-\dot{y}\right)+p^{2} z_{f}=h s \quad \dot{\phi} t,
$$

where:

$n=\frac{b_{2}}{2 m}$ is the Maxwell model damping factor

$p=\sqrt{\frac{k_{1}}{m}}$ - natural pulsation (angular frequency) of the $1 \mathrm{DOF}$ mechanical system with Hooke model (without Maxwell model)

$\checkmark \zeta=\frac{b_{2}}{b_{c} r}=\frac{b_{2}}{2 \sqrt{m_{2} k}}$ - damping ratio of the 1DOF mechanical system with Maxwell model (without Hooke model)

$\Omega=\frac{\omega}{p}$ - relative pulsation

Considering the second equation of (1), the mathematical model of the mechanical system can be written as follows [7]: 


$$
m \ddot{z}+k_{2} y+k_{1} z_{f}=F_{O} s \quad \dot{\omega} t
$$

THE ANALYSIS OF THE FORCED VIBRATION AMPLITUDE OF THE MECHANICAL SYSTEM

The 1DOF mechanical system has two elastic linear elements (Hooke model) and one viscous elastic element (Newton model), therefore all the displacements have harmonic time variation with different phase shifts to harmonic force $F(t)=H$ s i root

$$
\begin{aligned}
& z_{f}(t)=A_{f} s\left(\omega t-\varphi_{0}\right) \\
& y(t)=A_{Y} s \quad i(\omega t-\alpha)
\end{aligned}
$$

$A_{f}$ - mass $\mathrm{m}$ displacement amplitude $A_{Y}$ - point $\mathbf{P}$ displacement amplitude $\varphi_{0}$ - phase shift $Z_{f \leftrightarrow F}$ $\alpha$ - phase shift $y_{\leftrightarrow} F$

Considering harmonic time variations functions (5) and (6), the moving differential equation becomes:

$$
\begin{aligned}
-m \omega^{2} s\left(\omega i t-\Phi_{0}\right)+k_{2} A_{Y} s & (\omega i t-\mu)+ \\
+k_{1} A s \quad\left(\omega t-\phi_{0}\right)=F_{0} s & \omega \dot{t} \quad n
\end{aligned}
$$
$\mathbf{P}$ is:

The dynamic balance of forces in the point

$$
\begin{aligned}
b_{2} \mid \omega A_{f} c & \left.\left(\omega t-\varphi_{0} s\right)-\omega A_{Y} c \quad(\omega t-\alpha)\right\}= \\
& =k_{2} A_{Y} s \quad(\omega i-1 \alpha)
\end{aligned}
$$

Solving the trigonometric equations (7) and (8) in $\left(A_{f}, \varphi_{0}, A_{Y}, \alpha\right)$, the amplitude of the forced vibration of the mass $m$ can be written

$$
A_{f}=\frac{F_{0}}{k_{1}} \cdot A(\Omega, \zeta, N)=A_{S} \cdot A(\Omega, \zeta, N),
$$

where:

$$
A_{s t}=\frac{F_{0}}{k_{1}} \text { is the static displacement (Hooke }
$$
model, without Maxwell model)

$$
A(\Omega, \zeta, N) \text { - amplitude factor }
$$

$$
A(\Omega, \delta, N)=\sqrt{\frac{1+\left(\frac{2 \zeta \Omega}{N}\right)^{2}}{\left(1-\Omega^{2}\right)^{2}+\left(N+1-\Omega^{2}\right)^{2}\left(\frac{2 \zeta \Omega}{N}\right)^{2}}}
$$

\section{AMPLITUDE FACTOR DIAGRAMS}

The relation (10) can be used to draw the amplitude factor $A$ diagrams function of the relative angular frequency $\Omega$, for different values of $\zeta$ and $N$.

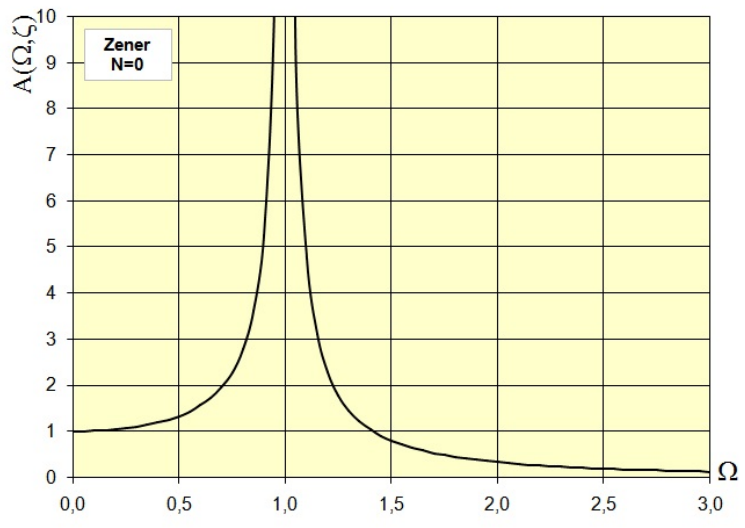

Fig. 6. Amplitude factor diagram SLS model - $\mathrm{N}=0$ ( $\mathrm{H}$ model)

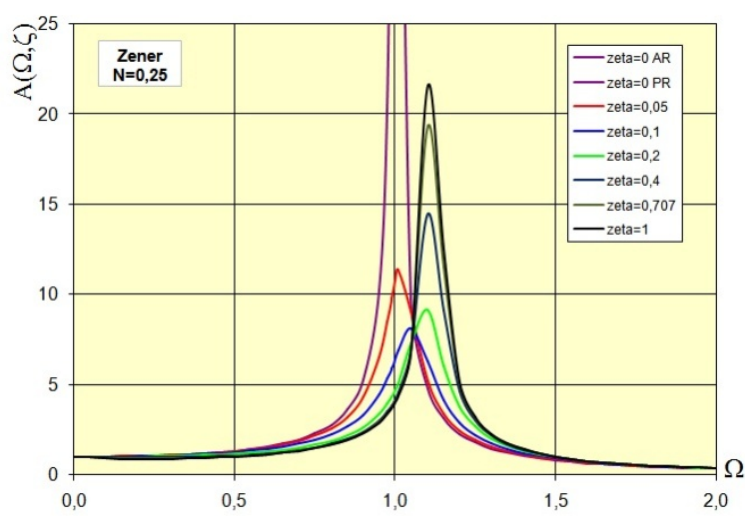

Fig. 7. Amplitude factor diagram SLS model - $\mathrm{N}=0.25$ 


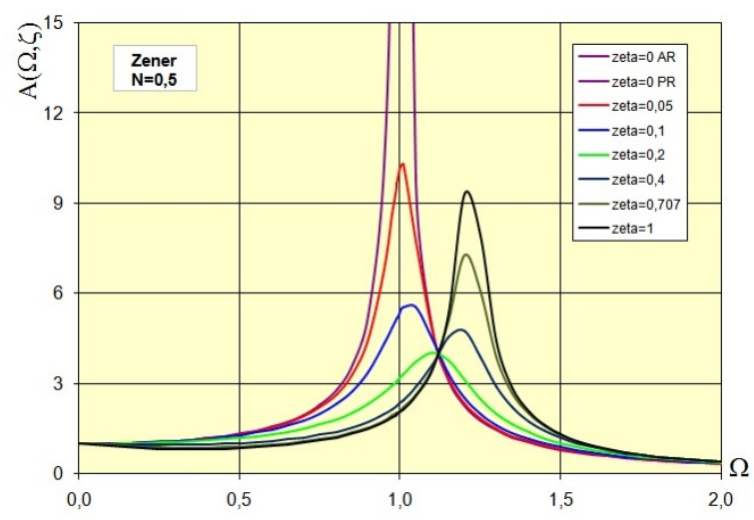

Fig. 8. Amplitude factor diagram SLS model - $\mathrm{N}=0.5$

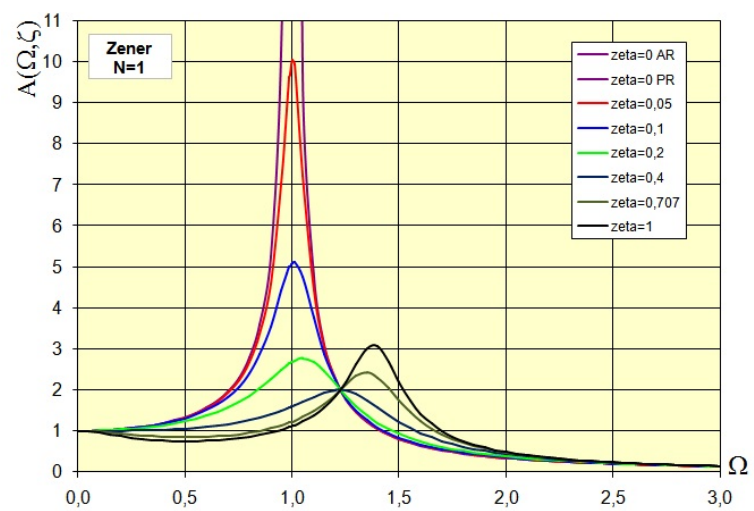

Fig. 9. Amplitude factor diagram SLS model - $\mathrm{N}=1$

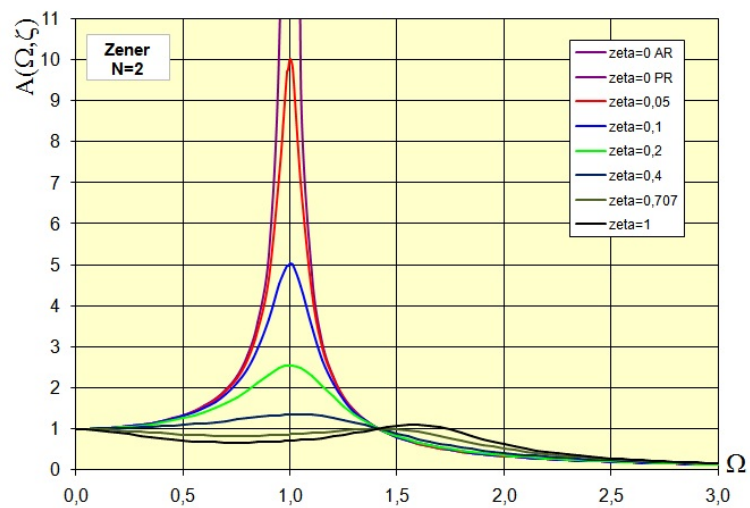

Fig. 10. Amplitude factor diagram SLS model - $\mathrm{N}=2$

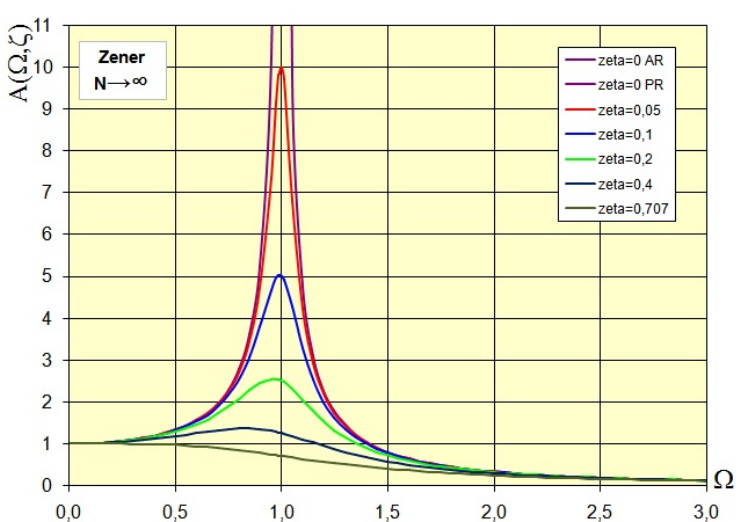

Fig. 11. Amplitude factor diagram SLS model - $\mathrm{N} \rightarrow \infty(\mathrm{V}-\mathrm{K}$ model $)$

\section{CONCLUSIONS}

for $N=0 / k_{2}=0$ (cancellation of elastic element from Maxwell model) or $\zeta=0$ (cancellation of viscous element from Maxwell model), SLS model becomes Hooke model; the amplitude factor diagram is shown in figure 6 and the amplitude resonance point is $\Omega=1$; the amplitude factor formula is as follows:

$$
A_{N=0}(\Omega) \equiv A_{\zeta=0}(\Omega)=\frac{1}{\left|1-\Omega^{2}\right|}
$$

for $N \rightarrow \infty / k_{2}>>k_{1}$ (the spring from Maxwell model is replaced by a rigid connection,), SLS model becomes a Voigt-Kelvin model; the diagram is shown in figure 11; the amplitude factor formula is:

$$
A_{N \rightarrow \infty}(\Omega, \zeta)=\frac{1}{\sqrt{\left(1-\Omega^{2}\right)^{2}+4 \zeta^{2} \Omega^{2}}}
$$

for Voigt-Kelvin model, the amplitude resonance point is $\Omega \leq 1$, see figure 11 ; considering a viscous damping $\zeta \neq 0$, the amplitude factor at resonance is:

$$
A_{V-K}^{r} \underset{e}{e}(\zeta)=\frac{1}{2 \zeta \sqrt{1-\zeta^{2}}}
$$

for SLS rheological model, the amplitude resonance are for $\Omega>1$; the value of the amplitude factor depends on the elasticity coefficients ratio $N$ (figures 7 to 10 ).

\section{REFERENCES}

[1] Flugge, W., Viscoelasticity, Springer, New York, 1975

[2] Christensen, R.M., Theory of Viscoelasticity, Dover Publications Inc., New York, 2010. 
[3] Dragan, N., Dynamic analysis of the parameters of the mechanical systems with structural damping. Viscoelastic SLS model. Part 1: Amplitude factor, The Annals of "Dunarea de Jos" University of Galati, Fascicle XIV Mechanical Engineering, ISSN 12245615, Galati, 2016

[4] Dragan, N., Dynamic analysis of the parameters of the mechanical systems with structural damping. Viscoelastic SLS model. Part 2: Transmissibility factor and isolation degree, The Annals of "Dunarea de Jos” University of Galati, Fascicle XIV Mechanical
Engineering, ISSN 1224-5615, Galati, 2016

[5] Spanu (Stefan), G.C., Dragan, N., Analysis of the transmissibility ratio and the isolation degree of the vibration for mechanical systems with structural damping. Zener model, Synthesis of Theoretical and Applied Mechanics, ISSN 2068-6331, Bucharest, 2017

[6] Bratu, P., Elastic Systems Vibrations, Technical Publishing House, Bucharest, 2000.

[7] Bratu, P., Bearing Elastic Systems for Machines and Equipment, Technical Publishing House, Bucharest, 1990. 